\title{
Effect of visual searching task on the postural stability of children
}

\section{CARINA L. MOREIRA ${ }^{1,2}$ | LEANDRO F. DE OLIVEIRA ${ }^{1}$ | MATHEUS B. BRITO1,2 | SÉRGIO T. RODRIGUES 1,2 | PAULA F. POLASTRI $\left.\right|^{1,2}$}

1 UNESP - Sao Paulo State University, Department of Physical Education, Faculty of Sciences, Bauru, São Paulo, Brazil.

2 Post-graduation Program in Movement Sciences, São Paulo State University - UNESP, São Paulo, Brazil.

Correspondence to: Paula Fávaro Polastri - Laboratory of Information, Vision and Action, Department of Physical Education, Faculty of Sciences, São Paulo State University, Bauru, São Paulo, Brazil. Address: Av. Eng. Luiz Edmundo Carrijo Coube, 14-01, Vargem Limpa, Bauru São Paulo, Brazil. Postal code:17033-360 Phone: +551431036082 .

email: paula.polastri@unesp.br

https://doi.org/10.20338/bjmb.v15i2.201

HIGHLIGHTS

- The relationship between gaze and postural control changes with age.

- The greater the attentional/cognitive demand for the tasks, the greater the CoP displacement.

- Challenging basis of support interfere with eye movement effects on children's body sway.

- The gaze performance of children differs from adults and between visual tasks.

- Children must develop the ability to modulate both the gaze and postural control.

\section{ABBREVIATIONS}

AlOs Areas of interest

AP Anterior-posterior

CNS Central nervous system

CoP Center of pressure

ML Medial-lateral

\section{PUBLICATION DATA}

Received 28092020

Accepted 25112020

Published 01062021

\begin{abstract}
BACKGROUND: The relationship between gaze behavior and postural stability under distinct cognitive loads is still unclear in children.

AIM: This study focuses on eye movements' effect on the children's postural stability by varying the visual tasks' attentional/cognitive demands.

METHOD: Twenty young adults (23.4 years-old, \pm 2.1 ) and sixteen children (10.3 years-old, \pm 0.4$)$ stand in semitandem position on a force plate, wearing a head-mounted eye-tracking while performing three 40 second-trials in the following order: stationary gaze task; free-viewing task; and visual searching task.

RESULTS: Children showed a higher number of fixations during the stationary gaze task $(p<0.0001)$, but this number was lower during free-viewing $(p<0.004)$ and visual searching tasks $(p<0.0001)$ when compared to young adults. Total fixation duration was lower in children than in young adults $(p<0.0001)$ and higher during stationary gaze task than free-viewing $(p<0.0001)$ and visual searching tasks $(p<0.0001)$ for both groups.

CONCLUSION: These results indicate that the performance of the visual searching task during the maintenance of a challenging stance may deteriorate children's postural stability suggesting that the relationship between gaze behavior and postural control is not well developed around the age of 10 years.
\end{abstract}

KEYWORDS: Eyes movements | Development | Postural control | Working memory | Gaze performance

\section{INTRODUCTION}

Visual information is used by the central nervous system (CNS) to adjust the motor commands to environmental changes to maintain a stable body position relative to the visual scene. Acquiring visual information depends on different gaze behaviors, such as gaze fixation, smooth pursuit, and saccadic eye movements. Performing saccades is crucial to promote an active search for relevant information in the environment while maintaining the eyes relatively stable (gaze fixation) is necessary to bring high-resolution visual information onto the fovea. ${ }^{1}$ Various cortical and subcortical structures underlie the reflexive (e.g., vestibular-ocular reflex, optokinetic nystagmus) and the voluntary control of eye movements, which are linked to head, neck, and body movements. ${ }^{2}$

It is known that both postural and oculomotor control develops through childhood into adolescence. Developmental changes in the dynamic relationship between visual information and body sway, for instance, is revealed in the reduction of body sway of 4-yearold children, following an improvement with age to achieve adult-like behavior around 12

\begin{tabular}{l|l|l|l|ll}
\hline Moreira et al. & 2021 & VOL.15 & N.2 & https://doi.org/10.20338/bjmb.v15i2.201
\end{tabular}


years old. ${ }^{3,4}$ Regarding the ability to control the eyes, it has been shown that gaze fixation, smooth pursuit, and saccadic eye movements are observed early in life and continue developing up to 15 years of age and later. ${ }^{5}$ Irving and colleagues ${ }^{6}$ showed that saccadic latency decrease from 3 to 14 years of age, presenting relative stability up to 50 years and a gradual increase at 80 years of age.

Recent studies have indicated an interaction between gaze and postural control, and it changes with age. ${ }^{7-9}$ Ajrezo and colleagues ${ }^{8}$ showed a reduction in children's body sway from 5 to 18 years old during the saccadic eyes movement task compared to the gaze fixation task. The authors also reported a decrease in the saccadic latency and enhancement in fixation quality with age. Contrariwise, Schärli and colleagues ${ }^{7,10}$ found that 5 years-old children increased the body sway during saccades task compared to older children and adults while standing in a narrow stance. These results were attributed to the immaturity of head control during the gaze shifts in such a challenging balance stance. ${ }^{7}$ Unfortunately, the results of gaze behavior were not reported in this study. In another study ${ }^{10}$ of these authors, it was observed a developmental improvement in children aged 6-12 years on body and head stabilization in space during gaze fixation as well as in free-viewing visual task. The larger body and head displacements were associated with larger gaze shifts in younger children during exploratory gaze tasks. ${ }^{10}$

Characteristics and complexity of visual and postural tasks should be considered when interpreting these results. Studies with young and older adults have demonstrated that performing complex oculomotor tasks leads to greater postural stability but can be affected by the stances' challenges. ${ }^{11-14}$ Also, Legrand and colleagues ${ }^{9}$ examined the interference between the oculomotor and postural tasks in 7-8-years-old children by manipulating the attentional/cognitive demands of visual tasks (fixation/prosaccades/antisaccades) and complexity of the postural tasks (standard/tandem Romberg stance). The results indicated longer latencies during the antisaccades task (the most difficult visual task) in children than in young adults when this visual task was performed with tandem Romberg stance (the most difficult postural task). These results are in line with the study by Polastri and colleagues ${ }^{12}$ for older adults who showed higher latency variability only during the vertical saccades task when they were standing on a semi-tandem basis of support. Altogether, these studies indicate that both the children and older adults presented increased body instability during the most challenging visual and postural tasks. Despite these findings, the relationship between gaze and postural control of children is still unclear concerning the reciprocal effects on visual and postural tasks. Our study adds novelty to the current understanding of the linkage between postural and oculomotor responses in children.

The current study aimed to investigate the effect of the attentional/cognitive demands of visual tasks on the children's postural stability during a challenging upright stance. We examined sway measures and eye movements recording during unrestricted free-viewing and searching visual tasks while children and adults were standing in a semitandem position, a challenging postural stance. First, we explored whether children's postural control would interact with the complexity of visual tasks in a more natural gazing context, allowing comparison with systematic gaze shifts usually employed by other studies.

Second, we increased the attentional/cognitive demands by using a visual searching task, followed by progressively less demanding free-viewing and stationary gaze tasks. ${ }^{13,15}$ Lastly, as many factors influence the visual attention and the selection of what is

\begin{tabular}{l|l|l|l|ll}
\hline Moreira et al. & 2021 & VOL.15 & N.2 & https://doi.org/10.20338/bjmb.v15i2.201
\end{tabular}


seen in the visual scene account for the eye movements consistency, ${ }^{16}$ we controlled stimulus salience and semantic relevance features of the visual images, ${ }^{17}$ the so-called bottom-up and top-down respective aspects, during the visual searching task. It has been assumed that the top-down stimulus requires higher-order processing, which demands more attentional efforts. ${ }^{18,19}$ Donnelly and colleagues ${ }^{19}$ pointed that $6-7$ years-old children present a lower capacity to perform visual search tasks guided by top-down stimulus than 8-10 years-old children and suggested that attentional components required by visual searching seem to develop from mid to late childhood.

We hypothesized that: 1) Children's postural sway would be increased during visual searching task compared to free-viewing and stationary gaze tasks, and they would be more unstable than young adults in all visual tasks; 2) Children would present lower number and longer duration of fixations than young adults in exploratory visual tasks; 3) Children would present higher number and shorter duration of fixations combined with higher postural sway during visual searching task compared to the free-viewing task.

\section{METHODS}

\section{Participants}

Twenty young adults from 20 to 29 years (mean age $=23.4, \pm 2.1$ years) and sixteen children from 9 to 11 years (mean age $=10.3, \pm 0.4$ years) participated in the study. Written consents were signed by the young adults and the children's parents allowing them to participate in the study. The local University Ethics Committee approved the experimental procedures according to the principles of the Declaration of Helsinki. None of the participants reported diagnosed neurological diseases, musculoskeletal problems, vestibular disorders, or visual impairments, which could compromise the experimental conditions' performance.

\section{Procedures}

Participants were required to stand in a semi-tandem position as still as possible on a force plate (AMTI - AccuGait), wearing a head-mounted eye-tracking (Applied Science Laboratory - ASL, model H6, USA), and looking straight ahead towards an image that was displayed on a screen fixed on the wall, placed at 2 meters, in front of them. The projector used to display the images was fixated on the ceiling and adjusted to the participant's height. The semi-tandem stance (dominant foot aligned ahead and medial to the non-dominant foot) was chosen to challenge the participant's postural stability in the medial-lateral direction $(\mathrm{ML})$.

Each participant performed three 40 second-trials in the following order: 1) Stationary gaze task - the participants were required to fixate a stationary target displayed on the center of the screen at eye-height. The target was a filled circle in red with $2 \mathrm{~cm}$ of diameter in white background (Figure 1-A); 2); Free-viewing task - the participants were required to look at the details of an image displayed on the screen, without any other instructions; 3) Visual searching task - the participants were instructed to look at the image projected previously on the screen and to locate specific changes in the features of the scene, reporting them at the end of the trial. This visual task required to recall the details of the image displayed during the free-viewing task. In this condition, two images were created to account for the influence of bottom-up and top-down factors on the visual searching ${ }^{17}$ (Figure 
1-C and 1-D). Half of each group's participants (ten young adults and eight children) looked only at the image with bottom-up changes while the other half of them looked only at the image with top-down changes. Figure 1 depicts the images projected on the screen during the stationary target condition (A), free-viewing condition (B), and visual searching condition with the locations of bottom-up (C) and top-down (D) changes. The bottom-up changes are located in the wall painting and the carpet (Figure 1-C), and the top-down changes are located in the tea-cup and lampshade (Figure 1-D). Participants were instructed to fixate the target or visually explore the images on the screen with eye movements only without move the head. There was no rest period between trials. However, they were instructed to request intervals between trials if necessary.

A

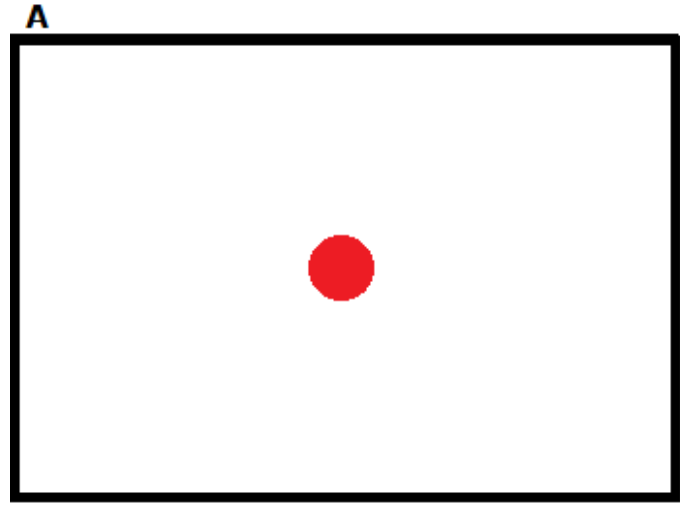

C

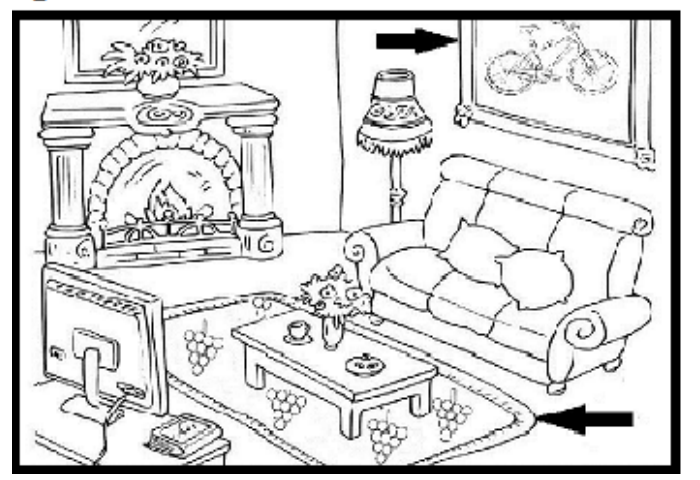

B

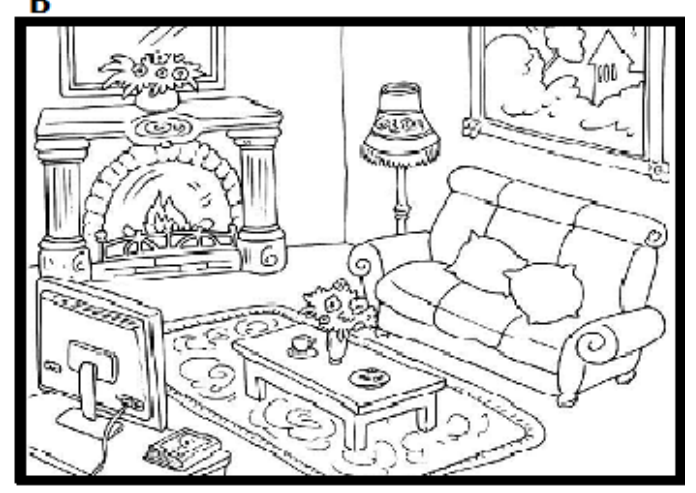

D

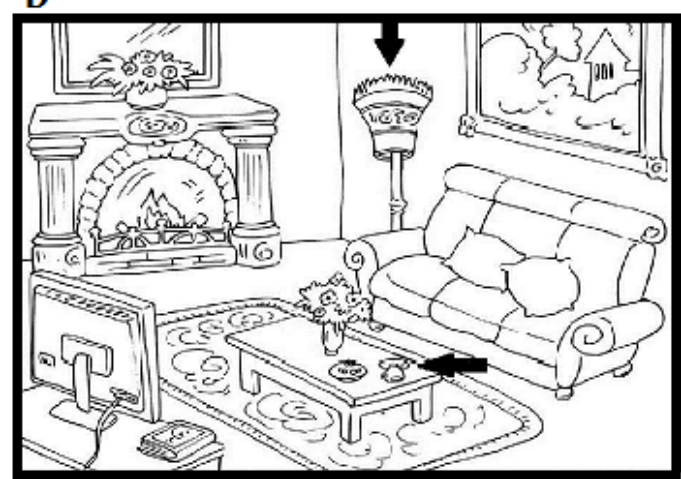

Figure 1. Representative images were displayed to the participants during the experimental procedures. Three trials were performed in the following order: stationary gaze task (panel $A$ ) with a filled circle in red displayed in white background; free-viewing task (panel B); and visual searching task (panels $C$ and D). Note: In the third trial (visual searching condition), half of the participants (ten young adults and eight children) looked only at the image $(C)$ with Bottom-Up changes in the wall painting and the carpet, and the other half of the participants looked only at the image (D) with Top-Down changes in the tea-cup and the lampshade. The arrows indicate the locations of the changes and were not displayed to the participants during the visual searching condition.

\section{Data analyses}

Center of pressure (CoP) displacement at anterior-posterior (AP) and $\mathrm{ML}$ axes were calculated using the forces and moments ( $F x, F y, F z$, and $M x$, My Mz) acquired by the force plate, and provided information about the participant's body sway. A head-mounted eye- 
tracking system measured eye movements. The accuracy of the eye-tracking equipment is 1 degree $\left(1^{\circ}\right)$ of change in the line-of-gaze. Participants' eye movements were recorded using Head-Mounted Eye Tracker (model H6, Applied Science Laboratory, USA). This videobased analysis system of eye movements contains two micro-cameras, one that records the eye and another the scene, attached to a headgear that was anatomically adjusted to the participant's head. In the eye video, pupil and corneal reflection centroids were identified, and the vector between both is used to determine horizontal and vertical coordinates of eye position on-scene video. To calibrate the system, participants visually fixed nine points ( 3 by 3 grid) displayed in front of them while the referred centroids were registered (nine-point calibration method). A second low-pass Butterworth filter with a $4 \mathrm{~Hz}$ cut-off frequency was used to filter the CoP data. The sampling rate was $60 \mathrm{~Hz}$ for all equipment.

From the CoP trajectory data, the following dependent variables were calculated: mean sway amplitude (standard deviation of the trajectory after the average position was subtracted from the data points throughout the trial); mean velocity (the trajectory of the total sway divided by the total duration of the trial); and mean sway frequency (sum of the product of power spectrum and frequency divided by the total sum of the power spectrum), in the AP and ML directions, and sway area (an ellipse that contains $95 \%$ of the CoP signals dispersion).

From the eye movements, the following dependent variables were calculated: number of fixations (sum of the fixations throughout each trial) (units); mean fixation duration (s) (mean of the intervals between the fixation onset and fixation offset throughout each trial); and the total fixation duration (sum of the fixation duration throughout each trial). Fixation onset was set when one degree of visual angle in horizontal and vertical axes exceeded 100 ms; fixation offset was defined when the initial fixation value deviated by more than one degree of visual angle in horizontal and vertical axes. Lastly, ASL Plus Results software (version 1.8.2.18) was used to define four areas of interest (AOls) in the image visualized by the participant relative to the location of the bottom-up (painting and carpet) and top-down (lampshade and tea-cup) changes during the visual searching task. The number of fixations, total fixation duration, and mean fixation duration were calculated for each AOI and then averaged across groups to quantify the gaze shifts towards the image changes. The number of errors reported by the participants regarding the changes' location was also computed for each group.

\section{Statistical analyses}

Firstly, ANOVA was performed in each CoP parameters, separately, for each group to examine differences between the participants who looked at the image with bottom-up changes and the ones who looked at the image with top-down changes during the visual searching task. There were no differences between groups $(p>0.05)$ in these analyses. Therefore, repeated-measures two-way ANOVA (2 groups $\times 3$ tasks) were performed to compare each CoP and gaze parameters between groups and within-group task conditions. Another set of ANOVA was performed to examine differences between groups relative to the gaze performance at the AOls. Post hoc tests with Bonferroni adjustments were conducted for main effects. The significance level was $p<0.05$ (SPSS, 17.0).

\begin{tabular}{l|l|l|l|lr}
\hline Moreira et al. & 2021 & VOL.15 & N.2 & https://doi.org/10.20338/bjmb.v15i2.201
\end{tabular} 


\section{RESULTS}

\section{CoP displacement}

Figure 2 depicts the CoP parameters for each group, in all task conditions, at AP (left panels) and ML (right panels) directions. Statistical significance indicated that children showed greater sway area, higher mean sway amplitude, and faster mean velocity than young adults in all visual tasks. The main effects and interactions for the CoP parameters are presented in Table 1.

Table 1 - ANOVA results for main effects of Group, Task, and Group and Task interaction relative to the center of pressure (CoP) displacement parameters. Note: significant values are in bold $(p<0.05)$.

\begin{tabular}{|c|c|c|c|c|c|c|}
\hline \multirow{2}{*}{ Cop Parameters } & \multicolumn{2}{|c|}{ Task Effect } & \multicolumn{2}{|c|}{ Group Effect } & \multicolumn{2}{|c|}{ Interaction Group-Task } \\
\hline & F value & $p$ & F value & $p$ & F value & $p$ \\
\hline Mean Sway Amplitude AP (cm) & 7.361 & $p<0.001$ & 34.571 & $p<0.0001$ & 5.725 & $p<0.005$ \\
\hline Mean Sway Amplitude ML (cm) & 2.539 & $p>0.05$ & 16.551 & $p<0.0001$ & 0.059 & $p>0.05$ \\
\hline Mean Sway Frequency AP (Hz) & 1.238 & $p>0.05$ & 0.299 & $p>0.05$ & 1.462 & $p>0.05$ \\
\hline $\begin{array}{c}\text { Mean Sway Frequency ML } \\
(\mathrm{Hz})\end{array}$ & 0.640 & $p>0.05$ & 0.513 & $p>0.05$ & 1.746 & $p>0.05$ \\
\hline Mean Velocity AP (cm/s) & 4.239 & $p<0.02$ & 32.392 & $p<0.0001$ & 7.090 & $p<0.003$ \\
\hline Mean Velocity ML (cm/s) & 1.468 & $p>0.05$ & 8.564 & $p<0.01$ & 1.245 & $p>0.05$ \\
\hline Sway Area $\left(\mathrm{cm}^{2}\right)$ & 5.315 & $p<0.005$ & 36.526 & $p<0.0001$ & 2.809 & $p<0.05$ \\
\hline
\end{tabular}

For AP mean sway amplitude, post hoc tests for interaction between groups and tasks revealed that only children showed greater mean sway amplitude during the visual searching task as compared to free-viewing $(p<0.02)$ and stationary gaze task $(p<0.05)$. At $\mathrm{ML}$ direction, post hoc tests pointed out that children showed higher mean sway amplitude than young adults $(p<0.0001)$. However, there were no task effect or interaction between groups and tasks in the ML direction.

For AP mean velocity, post hoc tests for interaction between groups and visual tasks revealed that children swayed faster in all visual tasks than young adults $(p<0.0001)$. However, only children showed faster mean velocity during the visual searching task as compared to free-viewing $(p<0.02)$ and stationary gaze tasks $(p>0.001)$. ML mean velocity was higher in children than in young adults $(p<0.01)$. There were no task effects or interactions between groups and tasks $(p>0.05)$. For the sway area, post hoc tests for interaction between groups and tasks revealed that children showed higher sway magnitude than young adults $(p<0.001)$ in all visual tasks. However, only children showed a higher sway area during the visual searching task than the stationary gaze task ( $p>0.01)$. No differences were found for mean sway frequency at $\mathrm{AP}$ and $\mathrm{ML}$ directions $(\mathrm{p}>0.05)$. 

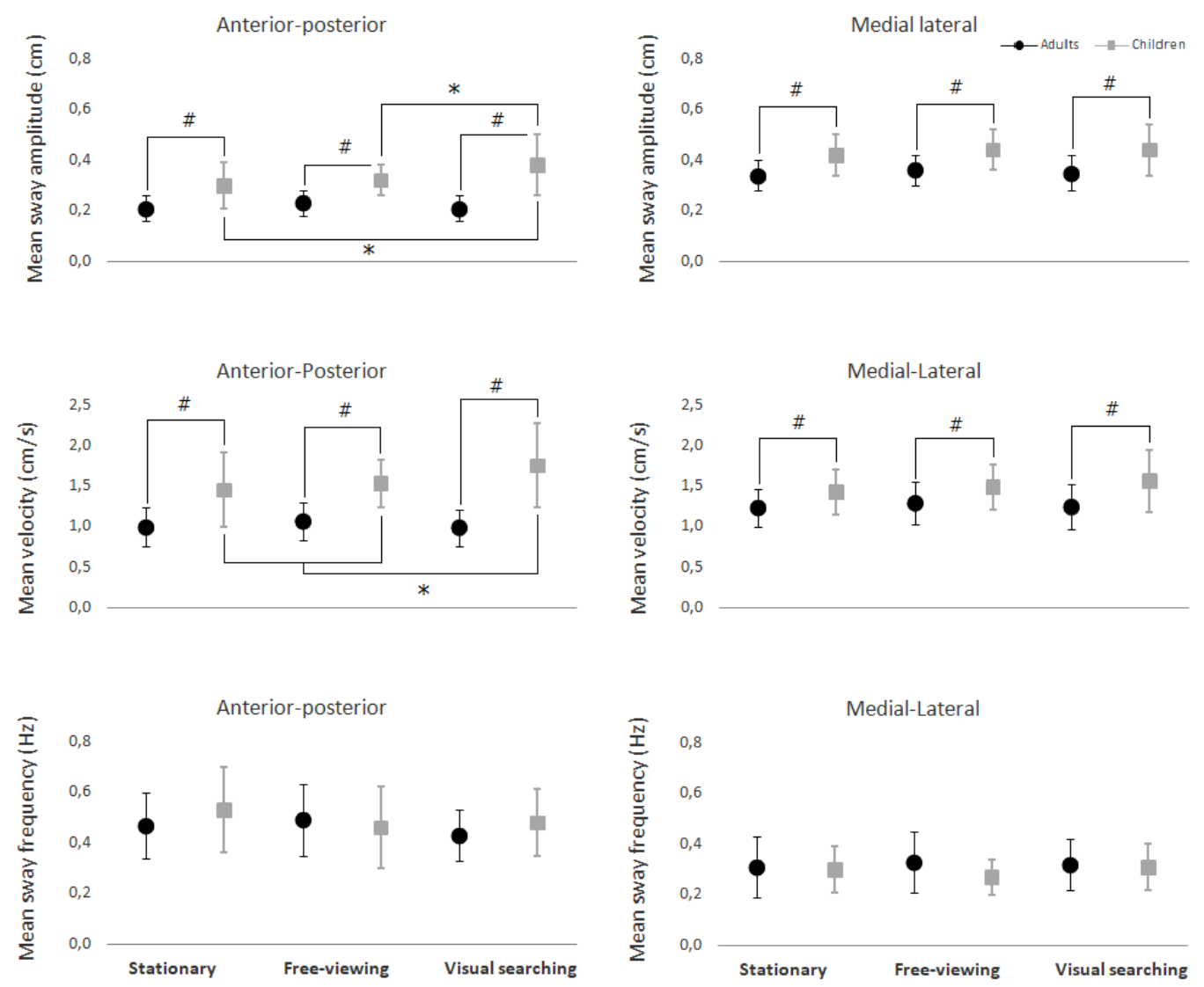

Figure 2. Mean and standard deviation values of mean sway amplitude, mean velocity, and mean sway frequency, at anterior-posterior (left panels) and medial-lateral (right panels) directions for each group (young adults and children) across visual tasks (stationary gaze, free-viewing, and visual searching tasks). Note: \# symbol indicates statistical significance for the main group effect; *symbol indicates statistical significance for groups and task interaction $(p<0.05)$.

\section{Gaze performance}

The main effects and interactions for the gaze variables are presented in Table 2. For the number of fixations, post hoc tests for interaction between groups and tasks revealed that young adults present a lower number of fixations in stationary gaze task $(p<0.004)$ and, on the other hand, a higher number of fixations during free-viewing $(p<0.004)$ and visual searching task $(p<0.0001)$ as compared to children. Young adults showed a lower number of fixations during stationary gaze than free-viewing $(p<0.0001)$ and visual searching tasks $(p<0.0001)$, and lower in free-viewing than in visual searching tasks $(p<0.02)$. Children showed a lower number of fixations during stationary gaze than free-viewing $(p<0.0001)$ and visual searching task $(p<0.0001)$. However, they did not show differences between freeviewing compared to visual searching task ( $p>0.05)$. 
Brazilian Journal of Motor Behavior

\section{Research Article}

Table 2 - Mean ( \pm standard deviation) values of gaze variables of young adults and children for each visual task condition. ANOVA results for main effects of Group, Task, and Group, and Task interaction. Note: Significant values are in bold $(p<0.05)$.

\begin{tabular}{|c|c|c|c|c|c|c|c|c|c|c|c|c|}
\hline \multirow{2}{*}{$\begin{array}{c}\text { Gaze } \\
\text { variables }\end{array}$} & \multicolumn{2}{|c|}{ Stationary } & \multicolumn{2}{|c|}{ Free-Viewing } & \multicolumn{2}{|c|}{ Visual Searching } & \multicolumn{2}{|c|}{ Task Effect } & \multicolumn{2}{|c|}{ Group Effect } & \multicolumn{2}{|c|}{ Interaction Group-Task } \\
\hline & Adults & Children & Adults & Children & Adults & Children & Fvalue & $p$ & Fvalue & $p$ & Fvalue & $p$ \\
\hline $\begin{array}{l}\text { Number of } \\
\text { Fixation } \\
\text { (units) }\end{array}$ & $\begin{array}{l}15.9 \\
(9.2)\end{array}$ & $\begin{array}{c}31.4 \\
(19.0)\end{array}$ & $\begin{array}{c}78 \\
(19.2)\end{array}$ & $\begin{array}{c}58.2 \\
(18.2)\end{array}$ & $\begin{array}{c}87.9 \\
(18.7)\end{array}$ & $\begin{array}{c}59.6 \\
(13.4)\end{array}$ & 114.302 & $p<0.001$ & 8.723 & $p<0.01$ & 20.441 & $p<0.001$ \\
\hline $\begin{array}{l}\text { Total Fixation } \\
\text { Duration (s) }\end{array}$ & $\begin{array}{l}39.4 \\
(2.5)\end{array}$ & $\begin{array}{l}31.7 \\
(9.3)\end{array}$ & $\begin{array}{l}24.0 \\
(3.6)\end{array}$ & $\begin{array}{l}18.3 \\
(6.3)\end{array}$ & $\begin{array}{l}25.9 \\
(3.4)\end{array}$ & $\begin{array}{l}17.5 \\
(6.3)\end{array}$ & 117.329 & $p<0.0001$ & 28.085 & $p<0.0001$ & 0.885 & $p>0.05$ \\
\hline $\begin{array}{c}\text { Mean } \\
\text { Fixation } \\
\text { Duration (s) }\end{array}$ & $\begin{array}{c}3.8 \\
(3.7)\end{array}$ & $\begin{array}{c}1.3 \\
(0.8)\end{array}$ & $\begin{array}{c}4.9 \\
(2.0)\end{array}$ & $\begin{array}{c}3.5 \\
(1.1)\end{array}$ & $\begin{array}{l}2.0 \\
(0.5)\end{array}$ & $\begin{array}{c}1.2 \\
(0.4)\end{array}$ & 15.641 & $p<0.0001$ & 10.252 & $p<0.004$ & 3.624 & $p<0.05$ \\
\hline $\begin{array}{l}\text { AOI Number } \\
\text { of Fixation } \\
\text { (units) }\end{array}$ & --- & ---- & ---- & ---- & $\begin{array}{c}58.5 \\
(12.0)\end{array}$ & $\begin{array}{c}44.9 \\
(12.5)\end{array}$ & --- & --- & 10.567 & $p<0.004$ & ---- & --- \\
\hline $\begin{array}{l}\text { AOI Total } \\
\text { Fixation } \\
\text { Duration (s) }\end{array}$ & --- & ---- & ---- & ---- & $\begin{array}{l}17.2 \\
(3.1)\end{array}$ & $\begin{array}{l}13.27 \\
(5.7)\end{array}$ & ---- & --- & 6.918 & $p<0.020$ & --- & ---- \\
\hline $\begin{array}{c}\text { AOI Mean } \\
\text { Fixation } \\
\text { Duration (s) }\end{array}$ & --- & --- & --- & ---- & $\begin{array}{c}1.2 \\
(0.2)\end{array}$ & $\begin{array}{c}1.1 \\
(0.2)\end{array}$ & ---- & --- & 0.780 & $p>0.05$ & ---- & ---- \\
\hline
\end{tabular}

For the total fixation duration, post hoc tests for the group effect indicated that children showed a lower fixation duration than young adults $(p<0.0001)$. Moreover, post hoc tests for task effect showed that participants presented a higher total fixation duration during stationary gaze task than free-viewing $(p<0.0001)$ and visual searching tasks $(p<0.0001)$. There was no difference between free-viewing and visual searching tasks $(p>0.05)$. There was no interaction between groups and tasks $(p>0.05)$. For mean fixation duration, post hoc tests for interaction between groups and tasks revealed that young adults showed higher mean fixation duration than children during stationary gaze $(p<0.02)$ and free-viewing tasks $(p<0.02)$ but not during the visual searching task $(p>0.05)$. For young adults, the mean fixation duration was lower during the visual searching task than the free-viewing $(p<0.0001)$ and stationary gaze tasks $(p<0.02)$. The mean fixation duration was higher for children during the free-viewing task compared to the stationary gaze $(p<0.03)$ and the visual searching task $(p<0.0001)$. Regarding the gaze shifts towards the AOls, post hoc tests for group effect indicated a higher number of fixations $(p<0.004)$ and higher total fixation duration $(p<0.02)$ for young adults as compared to children. No differences were found for mean fixation duration (see Table 2). One young adult and four children indicated incorrect locations for the images' changes during the visual searching task.

\section{DISCUSSION}

The current study aimed to investigate the effect of eye movements during unrestricted gaze behavior on the children's postural stability by varying the attentional/cognitive demands of the visual tasks while the participants were standing in a challenging stance. Our findings confirmed our hypotheses. Children showed greater and 
faster body sway, at AP direction, during the visual searching than the free-viewing and the stationary gaze tasks. They oscillated more than young adults in all visual tasks. Interestingly, children were also more unstable as compared to young adults in the ML direction. However, there were no differences between visual tasks, likely due to the effect of the challenging stance in the ML axis. These aspects will be discussed further.

The overall results indicated that eye movements through exploratory gaze behavior increased children's postural sway from 9 to 11 years compared to gaze fixation, ${ }^{10}$ instead of attenuating it as shown by previous studies during target-guided saccadic eye movements. ${ }^{8,9}$ It has been suggested that the postural control system's development relative to the head stabilization is the limiting factor to the postural stability in children.8,10 Schärli and colleagues ${ }^{10}$ demonstrated that young children's gaze-related head movements were associated with higher CoP displacement during an exploratory gaze condition (watching a movie) until, at least, the age of 12 years. In our study, participants were instructed to stand as still as possible and visually explore the image with eyes-only, without moving their heads. However, since the stimuli visual angle was not limited to 15 degrees ${ }^{20}$, to avoid the need for compensatory head movements, it is possible that children have increased the head movements and it has influenced the overall children's postural stability. Adults' postural sway was not affected by the gaze behavior corroborating this idea.

However, head instability cannot be the only factor to explain the increase in the children's body sway since the postural sway was dependent on the complexity of the visual task. We found that postural stability decreased as the attentional/cognitive demand required to perform the oculomotor tasks increased. It played an important role in the regulation of the postural control and gaze performance of the children. Indeed, our results suggest that the increase in the cognitive engagement to perform precise gaze shifts, along with the challenge imposed by the basis of support to maintain the upright position, exceeded the capacity limits of the CNS, which presumably reduced the efficacy of the efferent mechanisms (efference copy and re-afferences) ${ }^{20}$ in improving the children's postural stabilization.9,13 These findings are in line with other studies that had already demonstrated an interaction between the complexity of the postural and visual tasks and their effects on children and young adults' postural sway and gaze performance. ${ }^{9,10,21}$

Studies have shown that the programming and execution of eye movements and fine-tuning the postural adjustments while individuals are performing difficult visual and postural tasks require attentional resources, and it seems to result in more robust brain activation. ${ }^{1,22}$ For instance, cortical activations suggesting higher control has been shown during tandem versus standard Romberg tasks, ${ }^{23}$ and it appears to deteriorate the performance of a spatial working memory task. ${ }^{24}$ In the present study, the visual searching task required working memory and planned eye movements (gaze fixation and saccades) to locate the images' changes, increasing the demand for attention. This reflected in an increase of the body sway in children, differently at AP and ML axes, indicating that perturbation direction due to the basis of support interacts with the eye movements effect on the postural stability of children. ${ }^{9}$

Luna et al. ${ }^{5}$ reported that the ability to make memory-guided saccades appears to develop around mid-adolescence and continues into adulthood. In line with these results, we have found differences between children aged 9 to 11 years and young adults in the gaze performance. Children presented a lower number of fixations in the free-viewing and visual

\begin{tabular}{l|l|l|l|ll}
\hline Moreira et al. & 2021 & VOL.15 & N.2 & https://doi.org/10.20338/bjmb.v15i2.201
\end{tabular}


searching tasks than young adults. Young adults increased the number of fixations according to the visual task complexity (visual searching $>$ free-viewing $>$ stationary gaze). However, children showed such an increase only between visual searching and stationary gaze task. On the other hand, children presented a higher number of fixations during the stationary gaze task indicating a less stable gaze behavior in this condition compared to young adults. Moreover, children moved their eyes less often towards the AOls than young adults, regardless of whether they were looking at bottom-up and top-down features. These results indicate an immaturity of the oculomotor system of children to efficiently control the eyes under unrestricted gaze conditions. Therefore, age-related changes in postural and oculomotor control systems seem to interfere in a more effective eye-posture relation in children.

Our study presents some limitations. First, we instructed the participants to remain upright as still as possible in a challenging stance and visually explore an image with eyesonly without moving the head. However, we did not measure head movements during visual tasks. Although our COP measures are consistent with other studies, it would be interesting to evaluate the magnitude of the head movements and investigate whether changes in COP displacement are consistent with changes in head motion during unrestricted gaze behavior. It could also help to explain the differences in postural performance and gaze behavior between children and young adults. Second, we did not randomize the order of visual tasks. Randomization of the trial order would require different images during the free-viewing and visual searching tasks. To avoid choosing images with different levels of complexity (bottomup and top-down influences), which itself might change the eye movements consistency in young adults and children ${ }^{16}$ and interfere in the postural stabilization, we chose to keep the order of the trials for all participants. Besides that, there was a dependency between the free-viewing and visual searching tasks regarding the memory requirements. Our results regarding these aspects should be interpreted with caution.

\section{CONCLUSION}

In conclusion, our findings showed that eye movements during visual searching decrease children's postural stability. In particular, the greater the attentional/cognitive demand, the greater the CoP displacement. Interestingly, the challenging basis of support modulated the visual task effect on the body sway at the perturbation direction (e.g., ML). It suggests that a functional interaction between the oculomotor system and the postural control system is not yet developed at 9 to 11 years old. In everyday life, moving the eyes to relevant aspects of the environment while standing in different stances is crucial to the success of many motor tasks. Therefore, children must develop the ability to modulate eyes and body movements relative to each other according to each task's demands (postural and visual) to achieve an adult-like eye-posture relation. Future studies should manipulate other combinations between visual and postural tasks to better understand the relationship between eye movements and posture in children.

\section{REFERENCES}

\begin{tabular}{l|l|l|l|lr}
\hline Moreira et al. & 2021 & VOL.15 & N.2 & https://doi.org/10.20338/bjmb.v15i2.201
\end{tabular} 
Brazilian Journal of Motor Behavior

\section{Research Article}

1. Leigh RJ, Zee DS. The Neurology of Eye Movements. Fifth Edit. Oxford University Press. New York: United States of America; 2006. 1075 p

2. Proudlock FA, Gottlob I. Physiology and pathology of eye-head coordination. Prog Retin Eye Res. 2007;26:486-515. doi: 10.1016/j.preteyeres.2007.03.004

3. Polastri PF, Barela JA. Adaptive visual re-weighting in children's postural control. PLoS One. 2013;8(12):1-10. doi: 10.1371/journal.pone.0082215

4. Peterson ML, Christou E, Rosengren KS. Children achieve adult-like sensory integration during stance at 12-years-old. Gait Posture. 2006;23(4):455-63. doi:

10.1016/j.gaitpost.2005.05.003

5. Luna B, Velanova K, Geier CF. Development of eye-movement control. Brain Cogn. 2008;68(3):293-308. doi: 10.1016/j.bandc.2008.08.019

6. Irving EL, Steinbach MJ, Lillakas L, Babu RJ, Hutchings N. Horizontal saccade dynamics across the human life span. Investig Ophthalmol Vis Sci. 2006;47(6):2478-84. doi: 10.1167/iovs.05-1311

7. Schärli AM, van de Langenberg R, Murer K, Müller RM. The influence of gaze behaviour on postural control from early childhood into adulthood. Gait Posture. 2012;78-84. doi: 10.1016/j.gaitpost.2012.01.008

8. Ajrezo L, Wiener-Vacher S, Bucci MP. Saccades improve postural control: A developmental study in normal children. PLoS One. 2013;8(11):e81066. doi: 10.1371/journal.pone.0081066

9. Legrand A, Doré-Mazars K, Lemoine C, Nougier V, Olivier I. Interference between oculomotor and postural tasks in 7-8-year-old children and adults. Exp Brain Res. 2016;234(6):1667-77. doi: 10.1007/s00221-016-4565-0

10. Schärli AM, Van De Langenberg R, Murer K, Müller RM. Postural control and head stability during natural gaze behaviour in 6- to 12-year-old children. Exp Brain Res. 2013;227(4):523-34. doi: 10.1007/s00221-013-3528-y

11. Rodrigues ST, Aguiar SA, Polastri PF, Godoi D, Moraes R, Barela JA. Effects of saccadic eye movements on postural control stabilization. Motriz Rev Educ Fis. 2013;19:614-9. doi: 10.1590/S1980-65742013000300012

12. Polastri PF, Barbieri FA, Brito MB et al. Semi Tandem base of support degrades both saccadic gaze control and postural stability particularly in older adults. Neurosci Lett. 2019;705(1):227-34. doi: 10.1016/j.neulet.2019.03.004

13. Bonnet $\mathrm{CT}$, Baudry S. Active vision task and postural control in healthy, young adults: Synergy and probably not duality. Gait Posture. 2016;48:57-63. doi: 10.1016/j.gaitpost.2016.04.016

14. Bonnet CT, Szaffarczyk S, Baudry S. Functional Synergy Between Postural and Visual Behaviors When Performing a Difficult Precise Visual Task in Upright Stance. Cogn Sci. 2017;41(6):1675-93. doi: 10.1111/cogs.12420 
15. Bonnet CT, Davin T, Hoang JY, Baudry S. Relations between Eye Movement, Postural Sway and Cognitive Involvement in Unprecise and Precise Visual Tasks. Neuroscience. 2019;416:177-89. doi: 10.1016/j.neuroscience.2019.07.031

16. Franchak JM, Heeger DJ, Hasson U, Adolph KE. Free Viewing Gaze Behavior in Infants and Adults. Infancy. 2016;21(3):262-87. doi: 10.1111/infa.12119

17. Wright RD, Ward LM. Shifts of visual attention: An historical and methodological overview. Can J Exp Psychol Can Psychol expérimentale. 1994;48(2):151-66. doi: 10.1037/11961961.48.2.151

18. Neves EMDA, Gonzaga A, Slaets AFF. Atenção Visual Seletiva para Reconhecimento de Objetos e Análise de Cenas. Multiciência. 2000;4(1):26-44.

19. Donnelly N, Cave K, Greenway R, Hadwin JA, Stevenson J, Sonuga-Barke E. Visual search in children and adults: Top-down and bottom-up mechanisms. Q J Exp Psychol. 2007;60(1):120-36. doi: 10.1080/17470210600625362

20. Stoffregen TA, Bardy BG, Bonnet CT, Pagulayan RJ. Postural Stabilization of Visually Guided Eye Movements. Ecol Psychol. 2006;18(3):191-222. doi: 10.1207/s15326969eco1803_3

21. Olivier I, Palluel E, Nougier V. Effects of attentional focus on postural sway in children and adults. Exp Brain Res. 2008;185(2):341-5. doi: 10.1007/s00221-008-1271-6

22. Ettinger U, Ffytche $\mathrm{DH}$, Kumari $\mathrm{V}$ et al. Decomposing the neural correlates of antisaccade eye movements using event-related fmri. Cereb Cortex. 2008;18(5):1148-59. doi: 10.1093/cercor/bhm147

23. Ouchi Y, Okada H, Yoshikawa E, Nobezawa S, Futatsubashi M. Brain activation during maintenance of standing postures in humans. Brain. 1999;122(2):329-38. doi: 10.1093/brain/122.2.329

24. Kerr B, Condon SM, McDonald LA. Cognitive Spatial Processing and the Regulation of Posture. J Exp Psychol Hum Percept Perform. 1985;11(1):617-22. doi: 10.1037/00961523.11.5.617

Citation: Moreira CL, de Oliveira LF, Brito MB, Rodrigues ST, Polastri PF. Effect of visual searching task on the postural stability of children. BJMB. 2021. 15(2): 79-90.

Editors: Dr Fabio Augusto Barbieri - São Paulo State University (UNESP), Bauru, SP, Brazil; Dr José Angelo Barela São Paulo State University (UNESP), Rio Claro, SP, Brazil; Dr Natalia Madalena Rinaldi - Federal University of Espírito Santo (UFES), Vitória, ES, Brazil.

Copyright:@ 2021 Moreira, de Oliveira, Brito, Rodrigues and Polastri and BJMB. This is an open-access article distributed under the terms of the Creative Commons Attribution-Non Commercial-No Derivatives 4.0 International License which permits unrestricted use, distribution, and reproduction in any medium, provided the original author and source are credited.

Funding: This study was financed in part by the Coordenação de Aperfeiçoamento de Pessoal de Nível Superior Brasil (CAPES) - Finance Code 001. Post-graduation Program in Movement Sciences, São Paulo State University UNESP.

Competing interests: The authors have declared that no competing interests exist.

DOI: https://doi.org/10.20338/bjmb.v15i2.201 